\title{
Pictorial Blood Loss Assessment Chart for evaluating heavy menstrual bleeding in Asian women
}

\author{
Jennifer KY Ko *, Terence T Lao, Vincent YT Cheung
}

\section{A B S T R A C T}

Introduction: Heavy menstrual bleeding is a common gynaecological problem, but some women may prefer not to articulate their menstrual problems. The objective of this study was to evaluate the usefulness and acceptability of the Pictorial Blood Loss Assessment Chart (PBAC) as a selfscreening tool in evaluation of menstrual blood loss among Asian women in Hong Kong.

Methods: This prospective cohort study recruited 206 women from the general gynaecology ward and out-patient clinic: 118 had self-perceived heavy menstrual bleeding and 88 had self-perceived normal menstrual flow. Participants were asked to fill in the PBAC for one menstrual cycle.

Results: Compared with women who had selfperceived normal menstrual flow, women with selfperceived heavy menstrual bleeding had significantly higher total PBAC scores and numbers of flooding episodes, larger clot sizes and numbers, more days of bleeding, and lower haemoglobin levels. Receiver- operating characteristic curve analysis demonstrated good pairwise associations of self-perceived symptoms with PBAC score and haemoglobin level.

Conclusions: The PBAC can be used to differentiate self-perceived heavy and normal menstrual bleeding in Asian women in Hong Kong. It can also serve as an additional indicator of possible heavy menstrual bleeding to alert women of the need to seek early medical attention.

\begin{tabular}{l} 
Hong Kong Med J 2021;27:399-404 \\
\hline https://doi.org/10.12809/hkmj208743 \\
${ }^{1}$ JKY Ko *, MB, BS, FHAKM (Obstetrics and Gynaecology) \\
${ }^{2}$ TT Lao, MD \\
${ }^{1}$ VYT Cheung, FRCOG, FRCSC
\end{tabular}

${ }^{1}$ Department of Obstetrics and Gynaecology, The University of Hong Kong and Queen Mary Hospital, Hong Kong

2 Department of Obstetrics and Gynaecology, The University of Hong Kong (Honorary), Hong Kong

* Corresponding author: jenko@hku.hk

\section{New knowledge added by this study}

- The Pictorial Blood Loss Assessment Chart (PBAC) offers a semi-objective method for evaluation of heavy menstrual bleeding in women whose cultural backgrounds may cause reluctance in discussing their gynaecological or menstrual problems.

- More than $10 \%$ of women with self-perceived normal menstrual bleeding had PBAC scores $>100$, had anaemia, and/or required iron supplements.

- The best PBAC cut-off score (76) yielded a sensitivity of $93.2 \%$ and a specificity of $83.0 \%$ for predicting selfperceived heavy menstrual bleeding.

\section{Implications for clinical practice or policy}

- The PBAC may be useful as a self-screening tool for heavy menstrual bleeding among Asian women in Hong Kong, facilitating early medical evaluation of apparently asymptomatic women with unrecognised anaemia.

- Development of PBAC-containing mobile apps or websites may improve the usability of the PBAC in clinical and research settings.

- Localisation of the PBAC to include items encountered daily (such as 'tofu' or 'palm', rather than coins) could improve the usefulness of this tool.

- The PBAC may be useful for evaluation of responses to interventions during randomised controlled trials involving women with adenomyosis and uterine fibroids.

\section{Introduction}

The clinical decision regarding a need for treatment of menstrual bleeding relies on the patient's perception of flow amount and its effects on her physical, emotional, and social well-being. ${ }^{1}$ However, retrospective recall regarding the amount of menstrual flow in previous cycles is heavily influenced by a woman's subjective perception and is not always associated with the measured blood loss. ${ }^{2}$ The 'gold standard' approach for assessment of menstrual blood loss is the alkaline haematin method, which requires a woman to collect all soiled sanitary products for laboratory assessment ${ }^{2}$; however, this is a cumbersome non-hygienic impractical method outside the research setting.

The Pictorial Blood Loss Assessment Chart 
以月經失血圖作為評估亞洲婦女的大量經血情況 高嘉意、勞子僖、張煜棠

引言：大量經血是常見婦科問題，但部份女性不擅於表達甚至不察覺 她們的月經失調問題。本研究旨在評估月經失血圖（PBAC）作為香 港亞洲女性經血的自我篩查工具的效用性和可接受性。

方法：這項前瞻性隊列研究納入來自普通婦科病房和門診的206名女 性, 當中 118 例自我感受經血量大, 88 例自我感受經血量正常。參與 者被要求填寫一個月經週期的PBAC評估。

結果：與自我感受經血量正常的女性相比, 自我感受經血量多的女性 的PBAC總分和血崩發作次數顯著更高、血塊大小和數量更大、出血 天數更多, 以及血紅蛋白水平更低。接受者操作特性曲線分析表明自 我感受症狀與PBAC評分和血紅蛋白水平間存在良好的成對關聯。

結論：PBAC可用於區分香港亞洲女性自我感受的大量經血和正常經 血, 以及作為可能大量經血的額外指標, 提醒婦女需要及早就醫。
(PBAC) is a scoring system developed as a semiquantitative evaluation of menstrual blood loss, which considers the number of sanitary products used, the degree to which these products are soiled with blood, the number and size of blood clots passed, and the number of flooding episodes. ${ }^{3}$ The PBAC has been validated with the alkaline haematin method to diagnose heavy menstrual bleeding in several studies in other populations. ${ }^{3-5}$ Furthermore, the PBAC has been used as a measurement tool to evaluate menstrual blood loss in systematic reviews and randomised controlled clinical trials. ${ }^{6}$

In the clinical setting, it can be difficult for a physician to determine the amount and implication of menstrual flow in a patient reporting heavy menstrual bleeding. Menstruation is a taboo topic in many communities, including among Asian women in Hong Kong. ${ }^{7-10}$ Some women may prefer not to, or find it difficult or embarrassing to, articulate details regarding their menstrual problems. ${ }^{7-9}$ Furthermore, some women may be unaware of heavy menstrual bleeding.

The objective of this study was to evaluate the usefulness of the PBAC as a self-evaluation tool for heavy menstrual bleeding. Additionally, we sought to determine the acceptability of the PBAC and whether PBAC scores were associated with menstrual blood loss severity among Asian women in Hong Kong.

\section{Methods}

This prospective cohort study compared PBAC scores between women who presented with and without heavy menstrual bleeding. Women were recruited between November 2014 and January 2016 through the gynaecology ward or the general gynaecology out-patient clinic of a university-affiliated hospital.
They attended the out-patient clinic for routine follow-up or were admitted to the ward for elective or emergent treatment. Inclusion criteria included good general health, absence of other medical conditions which might lead to anaemia, no prior PBAC use, and age $\geq 18$ years. Women were excluded if they were pregnant, in menopause, receiving hormonal treatment, mentally incompetent, and/or undergoing treatment/monitoring of a gynaecological malignancy. Ethics approval was obtained from the Institutional Review Board of The University of Hong Kong/Hospital Authority Hong Kong West Cluster. Written informed consent was obtained from all study participants.

Women were approached by the research nurse and were placed into heavy menstrual bleeding and normal menstrual bleeding groups based on their self-reported menstrual cycle symptoms over the preceding 6 months. All group allocations were noted by the nurse. All participants, regardless of perceived menstrual flow, were instructed by the research nurse to fill in a PBAC for one cycle in the next cycle. They were also instructed to answer a question regarding whether they found the PBAC acceptable (yes/no) and a question regarding the ease of use of the PBAC (scale of 1-5; 1=easiest and $5=$ hardest). The PBAC originally described by Higham et $\mathrm{al}^{3}$ was used, but diagrams of clot sizes were modified to the sizes of local coins. The PBAC consisted of a series of diagrams representing lightly, moderately, and heavily soaked towels and tampons (depending on the degree of staining) to evaluate menstrual blood loss. ${ }^{3}$ The numbers of pads or tampons used each day were recorded. In the event of clot passage, the number and size were recorded; flooding episodes were also recorded. A total score was calculated by multiplying by a factor of 1 for each lightly soiled item, 5 for each medium soiled item, 10 for a fully soaked tampon, and 20 for a fully soaked pad. ${ }^{3}$ Small and large clots were given a score of 1 and 5, respectively. ${ }^{3}$ Women continued to use their own sanitary products (ie, products used prior to the study) and were asked to document the types and sizes of sanitary products used. Each woman was asked to return the completed PBAC to the research nurse by mail in a stamped envelope. The following clinical data were retrieved from the women's electronic medical records and used in the analysis: age, haemoglobin level within 3 months before the consultation or on the day of consultation (if available), and the iron supplement status (using/ not using).

The sample size was determined based on an anticipated $20 \%$ difference in accuracy endpoints between study groups and a standard deviation of $40 \%$. Allowing for $10 \%$ non-responders, the calculated sample size per group was 70 women. Statistical tests were performed using SPSS Statistics 
TABLE I. Reasons for presentation in women with self-perceived heavy and normal menstrual bleeding

\begin{tabular}{|c|c|c|c|}
\hline \multicolumn{2}{|c|}{ Self-perceived heavy menstrual bleeding $(n=118)$} & \multicolumn{2}{|c|}{ Self-perceived normal menstrual bleeding $(n=88)$} \\
\hline Diagnosis & No. (\%) & Diagnosis & No. (\%) \\
\hline Uterine fibroids & $90(76.3 \%)$ & Ovarian cyst & $30(34.1 \%)$ \\
\hline Adenomyosis & $10(8.5 \%)$ & Dysfunctional uterine bleeding & $13(14.8 \%)$ \\
\hline Dysfunctional uterine bleeding & $9(7.6 \%)$ & Uterine fibroids & $10(11.4 \%)$ \\
\hline Ovarian cyst & $5(4.2 \%)$ & Endometriosis & $10(11.4 \%)$ \\
\hline Endometrial polyp & $3(2.5 \%)$ & Infertility & $8(9.1 \%)$ \\
\hline \multirow[t]{6}{*}{ Hydrosalpinx } & $1(0.8 \%)$ & Endometrial polyp & $6(6.8 \%)$ \\
\hline & & Dysmenorrhoea & $5(5.7 \%)$ \\
\hline & & Pelvic inflammatory disease & $3(3.4 \%)$ \\
\hline & & Adenomyosis & $1(1.1 \%)$ \\
\hline & & Vaginitis & $1(1.1 \%)$ \\
\hline & & Screening for sexually transmitted disease & $1(1.1 \%)$ \\
\hline
\end{tabular}

(Windows version 24; IBM Corp, Armonk [NY], United States). Comparisons between groups were made using the Chi squared test for categorical variables and the non-parametric Mann-Whitney $U$ test for continuous variables. Continuous variables were expressed as median and range. A P value of $<0.05$ was considered statistically significant. The kappa statistic was used to test agreement between subjective evaluation of heavy menstrual bleeding and the PBAC score at various cut-off scores. Predictions of heavy menstrual bleeding according to the PBAC score and haemoglobin level were determined using area under the receiver-operating characteristic curve analysis.

\section{Results}

The response rate was better than expected and more women than expected were recruited in each clinic session; this yielded a final sample size larger than originally planned. However, among 292 women who were asked to complete the PBAC, the return rate was only 206/292 (70.5\%). In all, 118 women had self-perceived heavy menstrual bleeding and 88 women had self-perceived normal menstrual flow. Haemoglobin level data were available in 179/292 (61.3\%) women (116 in the heavy menstrual bleeding group and 63 in the normal menstrual bleeding group). Table 1 summarises the reasons for presentation in both groups of women. The PBAC scores based on different diagnoses are shown in Table 2. Women with heavy menstrual bleeding were older than women with normal menstrual bleeding (median age 44 years, [interquartile range $=40-48$ ] vs 38 years [interquartile range $=31-43$ ], respectively, $\mathrm{P}<0.001$ ). There was no significant difference in education level between groups (Table 3 ).
TABLE 2. Pictorial Blood Loss Assessment Chart scores in women with different diagnoses*

\begin{tabular}{lcc}
\hline Diagnosis & PBAC score & P value \\
\hline Uterine fibroids & $508(155-1125)$ & $<0.001$ \\
Adenomyosis & $991(96-4444)$ & \\
Endometrial polyp & $77(54-137)$ & \\
Dysfunctional uterine bleeding & $63(41-125)$ & \\
Others & $56(42-70)$ & \\
\hline
\end{tabular}

Data are shown as median (interquartile range), unless otherwise specified

Nearly all women in the study used pads; one woman used both pads and tampons. In total, 147/206 (71.4\%) women used various brands and sizes of pads with distinct absorbency characteristics during the menstrual cycle; the remaining 59/206 (28.6\%) women used only one type of pad. Seven women used diapers and three women used postpartum pads. The median PBAC scores of women who reported heavy and normal menstrual bleeding were 497 (interquartile range $=152-1112$ ) and 54 (interquartile range $=41-65$ ), respectively (Table 3 ). Compared with women who had normal menstrual flow, women with heavy menstrual bleeding had significantly higher total PBAC scores and numbers of flooding episodes, larger clot sizes and numbers, more days of bleeding, and lower haemoglobin levels (Table 3). Using cut-off scores of 76, 80,100,130, 150, and 185 , levels of agreement between PBAC score and self-reported symptoms in the diagnosis of heavy menstrual bleeding are shown in Table 4 . Women with anaemia, defined as haemoglobin level $<11.0 \mathrm{~g} / \mathrm{dL}$, had significantly higher median PBAC scores than did women without anaemia (508 [interquartile 
TABLE 3. Pictorial blood loss assessment chart (PBAC) score, haemoglobin level, days of bleeding, ease of use of the PBAC, and education level in women with selfperceived heavy and normal menstrual bleeding*

\begin{tabular}{lccr}
\hline & $\begin{array}{c}\text { Self-perceived heavy } \\
\text { menstrual bleeding } \\
(\mathbf{n = 1 1 8})\end{array}$ & $\begin{array}{c}\text { Self-perceived normal } \\
\text { menstrual bleeding } \\
\mathbf{( n = 8 8 )}\end{array}$ & P value \\
\hline Total PBAC score & $497(152-1112)$ & $54(41-65)$ & $<0.001$ \\
\hline Flooding & $90(20-190)$ & $0(0-0)$ & $<0.001$ \\
\hline Clots & $39(9-168)$ & $0(0-0)$ & $<0.001$ \\
\hline Pads & $375(96-736)$ & $48(38-59)$ & $<0.001$ \\
\hline Haemoglobin level & $8.6(7.2-10.1)$ & $11.6(11.0-12.1)$ & $<0.001$ \\
\hline Days of bleeding & $8(6-12)$ & $5(4-6)$ & $<0.001$ \\
\hline Ease of use ${ }^{\dagger}$ & $2(2-3)$ & $2(2-3)$ & 0.618 \\
\hline Iron supplements & $94(79.7 \%)$ & $12(13.6 \%)$ & $<0.001$ \\
\hline Education level & & & 0.363 \\
\hline Below primary & $2(1.7 \%)$ & 0 & \\
\hline Primary & $4(3.4 \%)$ & $1(1.1 \%)$ & \\
\hline Secondary & $74(62.7 \%)$ & $53(60.2 \%)$ & \\
\hline Tertiary & $38(32.2 \%)$ & $34(38.6 \%)$ & \\
\hline Data are shown & & & \\
\hline
\end{tabular}

* Data are shown as median (interquartile range) or No. (\%), unless otherwise specified In a scale of I to 5 ( I being the easiest and 5 the hardest)

TABLE 4. Levels of agreement between Pictorial Blood Loss Assessment Chart (PBAC) score and self-reported symptoms in the diagnosis of heavy menstrual bleeding*

\begin{tabular}{lccc}
\hline $\begin{array}{l}\text { PBAC cut-off } \\
\text { score }\end{array}$ & Sensitivity $(\mathbf{n = 1 1 8})$ & Specificity $(\mathbf{n = 8 8 )}$ & $\begin{array}{c}\text { Cohen's kappa } \\
\text { coefficient }(\kappa)\end{array}$ \\
\hline 76 & $110(93.2 \%)$ & $73(83.0 \%)$ & 0.770 \\
80 & $105(89.0 \%)$ & $74(84.1 \%)$ & 0.732 \\
100 & $95(80.5 \%)$ & $78(88.6 \%)$ & 0.679 \\
130 & $91(77.1 \%)$ & $81(92.0 \%)$ & 0.672 \\
150 & $90(76.3 \%)$ & $85(96.6 \%)$ & 0.703 \\
185 & $86(72.9 \%)$ & $88(100.0 \%)$ & 0.697 \\
\hline
\end{tabular}

Data are shown as No. (\%), unless otherwise specified

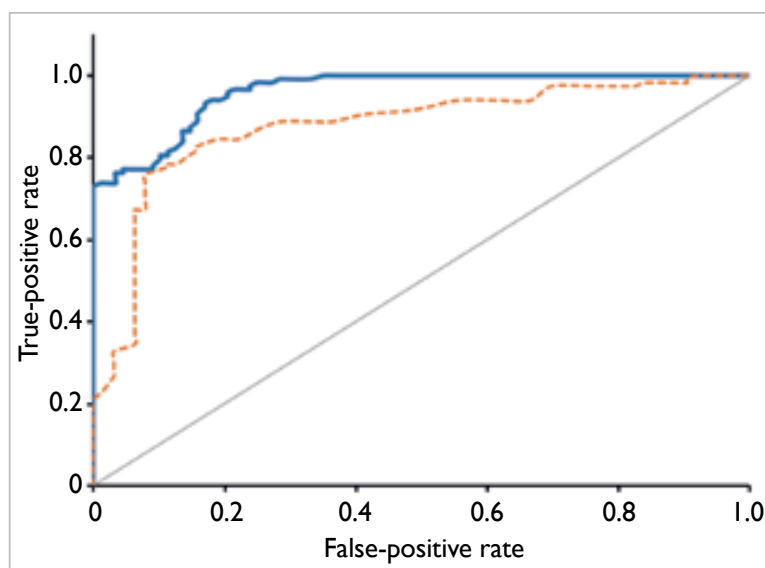

FIG. Receiver-operating characteristic curves demonstrating the predictive abilities of the pictorial blood loss assessment chart (blue line) and haemoglobin level (orange line) for heavy menstrual bleeding range $=168-1087$ ] vs 58 [interquartile range $=46-84$ ], $\mathrm{P}<0.01)$. Receiver-operating characteristic curves demonstrating the predictive abilities of the PBAC and haemoglobin level for heavy menstrual bleeding are shown in the Figure. The area under the receiveroperating characteristic curves of the PBAC and haemoglobin level for prediction of heavy menstrual bleeding were 0.961 (95\% confidence $=0.940-09.982$ ) and $\quad 0.876 \quad(95 \% \quad$ confidence $=0.821-0.931)$, respectively. The PBAC cut-off score with the highest Youden index was 76, which yielded a sensitivity of $93.2 \%$ and a specificity of $83.0 \%$ for predicting selfperceived heavy menstrual bleeding.

All women in our study were able to complete the PBAC. Missing information was filled in with the help of the research nurse via phone contact after return of the PBAC. Twenty-eight women (13.6\%) who began the PBAC on the day of consultation were contacted by phone to urge them to return the PBAC using the stamped envelopes. Another 11 women (5.4\%) with prolonged menstrual bleeding did not provide full details regarding their menstrual bleeding; they were contacted by phone for confirmation. In all, 200/206 women (97.1\%) found the PBAC acceptable: $113 / 118$ (95.8\%) in the heavy menstrual bleeding group and 87/88 (98.9\%) in normal menstrual bleeding group. Assuming that the reason for non-response was that those women found the PBAC to be unacceptable, the acceptability rate was $200 / 292(68.5 \%)$. There was no significant difference in the perceived ease of use of the PBAC; the median rating was 2 in both groups $(\mathrm{P}=0.618$; Table 3$)$. Notable written comments from the women concerning the PBAC were that it could not accurately describe their menstrual blood loss $(n=19)$, it required explanation $(n=11)$, it was inconvenient or involved recall problems $(n=3)$, and it did not record other symptoms which were more distressing $(\mathrm{n}=1)$.

\section{Discussion}

Our results suggested that the reported PBAC scores in this group of Asian women comprised a useful tool for differentiating self-perceived heavy and normal menstrual bleeding. Heavy menstrual bleeding considerably impacts a woman's quality of life; interventions should be designed to improve the quality of life, rather than focusing on the exact amount of menstrual blood loss. ${ }^{1}$ Nevertheless, some women may be unaware of heavy bleeding or find it difficult to describe the amount of menstrual flow. The PBAC offers a semi-objective method for initial self-evaluation of the amount of menstrual bleeding in women whose cultural backgrounds may cause reluctance in discussing their gynaecological or menstrual problems. This self-evaluation can alert women to seek medical attention, thus facilitating clinical evaluation and treatment. The PBAC cut-off 
scores included in Table 4 have been used in previous studies to imply heavy menstrual bleeding. ${ }^{3-5,11}$ The recommendation of a particular cut-off score depends on the clinical context (ie, whether a higher sensitivity or specificity is required). For example, if the PBAC is used as a screening tool, a lower cut-off score may be appropriate to alert women to seek medical attention. In contrast, if the PBAC is used to evaluate women with heavy menstrual bleeding for potential participation in a research study, a higher cut-off score may be used to recruit women with more severe symptoms to evaluate their response to treatment.

In our study, 10 women $(11.4 \%)$ in the selfperceived normal menstrual bleeding group had PBAC scores of $>100$, although they reported normal menstrual bleeding. In the self-perceived normal menstrual bleeding group, 14 women had anaemia (haemoglobin level $<11.0 \mathrm{~g} / \mathrm{dL}$ ), among which five women had a haemoglobin level of $<10.0 \mathrm{~g} / \mathrm{dL}$. Twelve women who reported normal menstrual bleeding were using iron supplements. Although most women accurately recognised heavy menstrual bleeding, use of the PBAC identified an additional $10 \%$ of women who might have unperceived abnormal bleeding. Of the 10 women with selfperceived normal menstrual bleeding (PBAC scores of 101-180), seven (70\%) had anaemia. Thus, use of the PBAC might enable identification of a small group of apparently asymptomatic women who had unrecognised anaemia, thereby facilitating earlier medical attention.

In our study, women were asked to use their own sanitary products, rather than using specific brands and sizes of pads; thus, our findings are more representative of realistic PBAC use, compared with results acquired in a research setting. Most women used different brands and sizes of pads with different absorbency characteristics, even within a single cycle. In addition, several women used adult diapers or postpartum pads, which implied substantial difference in blood loss compared with the usual sanitary pads. The range of PBAC scores was much larger in our study than in previous studies..$^{3-5,11,12}$ One woman in our study had a PBAC score of 32301 ; she had prolonged vaginal bleeding for 56 days and had a haemoglobin level of $4.5 \mathrm{~g} / \mathrm{dL}$. Women with adenomyosis and uterine fibroids had significantly higher PBAC scores than did women with other diagnoses. Therefore, the PBAC may be useful for evaluation of responses to interventions during randomised controlled trials involving these groups.

Although women in our study who returned the PBAC found it acceptable and generally easy to use, the return rate should be considered. Notably, $19 / 206$ (9.2\%) women commented that the range of icons in the PBAC did not accurately reflect their blood loss on pads or clots because they experienced difficulty in evaluating the amount of blood loss (based on a particular stain) when comparing among pads with different absorbency characteristics. The clots were of irregular size and women felt that a scale or use of items encountered daily (such as 'tofu' or 'palm', rather than coins) could more accurately describe these clots. Women (particularly in the heavy menstrual bleeding group) who had to sit on the toilet during flooding episodes could not quantify their bleeding; several women with prolonged bleeding did not continue the PBAC evaluation because they felt that continuing the documentation was time-consuming and annoying. In total, 5.3\% of the women commented that clearer instructions could be provided. This is consistent with the findings by Zakherah et $\mathrm{al}^{5}{ }^{5}$ who reported that improved instructions led to greater accuracy when a physician or nurse reviewed the documentation with the patient. The role of the nurse in our study was crucial. Our research nurse found it helpful to demonstrate to the women how to fill in the PBAC using their current or previous cycle; the nurse also helped the women to complete the PBAC in the event of substantial missing information, especially among women with prolonged menstrual bleeding. Some women probably completed the PBAC by recall, rather than in a day-by-day manner. This aspect should be considered when the PBAC is applied as a self-screening tool. The development of PBACcontaining mobile apps or websites accessible by the public may improve the usability of the PBAC as a self-screening tool in terms of better convenience and less recall bias, especially among younger women.

Our study had some limitations. First, we only evaluated use of the PBAC in a small group of patients who presented for clinical treatment, rather than the general population; this may limit the generalisability of the results. Second, we did not study the inter-cycle variability in PBAC score or the effects of other demographic factors (eg, household income) which may affect the use of the PBAC. Although only one cycle of menstrual bleeding was charted in our study and women may have unusual menstrual flow in subsequent cycles, previous studies have demonstrated high consistency with low inter-cycle variation in women who completed a second PBAC evaluation without treatment. ${ }^{11}$ Third, patients may have been offered treatment during the consultation; because the PBAC was completed in the cycle after consultation, the PBAC score may not fully reflect the pre-consultation reported symptoms, especially among women with self-perceived heavy menstrual bleeding. Fourth, compliance with iron therapy was not checked; this could have affected the haemoglobin results. However, the aim of our study was to evaluate the relationship between the PBAC 
score and self-perceived menstrual flow. Overall, the results of this population-specific study might support the use of the PBAC as a potential selfscreening tool for heavy menstrual bleeding among Asian women in Hong Kong.

There is considerable endpoint heterogeneity in the current literature with respect to the outcomes of various treatment options for heavy menstrual bleeding. Furthermore, there is currently no core outcome set for valid comparison and interpretation of data from research studies and assessments regarding abnormal uterine bleeding. ${ }^{6}$ Although PBAC scores have shown high interindividual variation, they had low intra-individual variation; ${ }^{11}$ thus, the PBAC may be useful in future studies of treatment responses in individual women. Despite the large variety of commercially available sanitary products, the PBAC remains a reliable screening tool for semi-quantitative evaluation of menstrual blood loss, which can alert women to seek medical attention for heavy menstrual bleeding. Additional studies are needed to confirm the clinical usefulness of the PBAC, especially in the context of the evolution and advancement of superabsorbent sanitary products currently available. Overall, the advantages of the PBAC are its relative objectivity and flexibility as a tool for screening, diagnosis, and evaluation of treatment effect.

\section{Author contributions}

Concept or design: JKY Ko, VYT Cheung. Acquisition of data: JKY Ko, VYT Cheung. Analysis or interpretation of data: All authors.

Drafting of the manuscript: JKY Ko.

Critical revision of the manuscript for important intellectual content: All authors.

All authors had full access to the data, contributed to the study, approved the final version for publication, and take responsibility for its accuracy and integrity.

\section{Conflicts of interest}

All authors have disclosed no conflicts of interest.

\section{Acknowledgement}

The authors thank Ms Wai-ki Choi for patient recruitment, teaching women about the Pictorial Blood Loss Assessment Chart, and managing the database.

\section{Declaration}

The study was presented in an oral presentation at the FOCUS in O\&G 2018 Congress in Hong Kong (17-18 November 2018).

\section{Funding/support}

This research received no specific grant from any funding agency in the public, commercial, or not-for-profit sectors.

\section{Ethics approval}

Ethics approval was obtained from the Institutional Review Board of The University of Hong Kong/Hospital Authority Hong Kong West Cluster (Ref: UW 14-299). Written informed consent was obtained from all study participants.

\section{References}

1. National Institute for Health and Care Excellence guideline. Heavy menstrual bleeding: assessment and management. 14 Mar 2018 (last updated 24 May 2021). Available from: http:// www.nice.org.uk/guidance/ng88. Accessed 25 Nov 2021.

2. Magnay JL, O’Brien S, Gerlinger C, Seitz C. A systematic review of methods to measure menstrual blood loss. BMC Womens Health 2018;18:142.

3. Higham JM, O'Brien PM, Shaw RW. Assessment of menstrual blood loss using a pictorial chart. Br J Obstet Gynaecol 1990;97:734-9.

4. Janssen CA, Scholten PC, Heintz AP. A simple visual assessment technique to discriminate between menorrhagia and normal menstrual blood loss. Obstet Gynecol 1995;85:977-82.

5. Zakherah MS, Sayed GH, El-Nashar SA, Shaaban MM. Pictorial blood loss assessment chart in the evaluation of heavy menstrual bleeding: diagnostic accuracy compared to alkaline hematin. Gynecol Obstet Invest 2011;71:281-4.

6. Herman MC, Penninx J, Geomini PM, Mol BW, Bongers MY. Choice of primary outcomes evaluating treatment for heavy menstrual bleeding. BJOG 2016;123:1593-8.

7. Garg S, Anand T. Menstruation related myths in India: strategies for combating it. J Family Med Prim Care 2015;4:184-6.

8. The Lancet Child Adolescent Health. Normalising menstruation, empowering girls. Lancet Child Adolesc Health 2018;2:379.

9. Agampodi TC, Agampodi SB. Normalising menstruation, empowering girls: the situation in Sri Lanka. Lancet Child Adolesc Health. 2018;2:e16.

10. Wong WC, Li MK, Chan WY, et al. A cross-sectional study of the beliefs and attitudes towards menstruation of Chinese undergraduate males and females in Hong Kong. J Clin Nurs 2013;22:3320-7.

11. Hald K, Lieng M. Assessment of periodic blood loss: interindividual and intraindividual variations of pictorial blood loss assessment chart registrations. J Minim Invasive Gynecol 2014;21:662-8.

12. Reid PC, Coker A, Coltart R. Assessment of menstrual blood loss using a pictorial chart: a validation study. BJOG 2000;107:320-2. 